\title{
Cutaneous polyarteritis nodosa causing refractory skin deformation and pigmentation as sequel*
}

\author{
Karin Okada ${ }^{1}$ \\ Hitoshi Mizutani ${ }^{1}$
}

\author{
Rina Nakamori ${ }^{1}$ \\ Keiichi Yamanaka ${ }^{1}$
}

DOI: http:/ /dx.doi.org/10.1590/abd1806-4841.20175696

\begin{abstract}
A 39-year-old woman presented with prominent and painful livedo reticularis lesions spreading on her upper and lower extremities. Histopathologically, the small- to medium-sized arteries in the deep dermis and subcutis showed necrotizing vasculitis with cellular infiltration, suggesting cutaneous polyarteritis nodosa. The serum levels of inflammatory markers normalized with aspirin $100 \mathrm{mg}$ /day and prednisolone $10 \mathrm{mg} /$ day within 2 months, and there was no other skin or organ involvement over 18 months of follow up. However, serious refractory skin depressions and pigmentation remained after two years of treatment. This suggests the importance of early and aggressive therapy for cutaneous polyarteritis nodosa to prevent unsightly skin sequel, as well as control of disease activity.
\end{abstract}

Kewbords: Pigmentation; Polyarteritis nodosa; Vasculitis

\section{INTRODUCTION}

Cutaneous polyarteritis nodosa (CPAN) is a rare form of vasculitis, which affects small- to medium-sized arteries in the dermis and subcutis. CPAN was reported by Lindberg in 1931 as a form of polyarteritis nodosa (PAN) limited to the skin. ${ }^{1}$ The common clinical features of CPAN are multiple subcutaneous nodules, livedo reticularis and small deep skin ulcers affecting the lower extremities.

The specific pathogenesis of CPAN is still unclear. Systemic corticosteroids are effective to patients with active inflammations. Anticoagulation or antiplatelet therapies are indicated for cases with arterial obstruction by thrombi, in addition to corticosteroid. We present a case with prominent livedoid skin lesions without ulceration, which rapidly responded to oral aspirin and low dose prednisolone. However, serious refractory skin depressions and pigmentation remained as sequel, suggesting the importance of early and aggressive therapy.

\section{CASE REPORT}

A 39-year-old woman consulted at our clinic complaining of painful multiple subcutaneous nodules with net-like appearance on her extremities for one year (Figure 1). Her vital signs were within normal limits. Weight loss, fatigue, oral ulcers, neurological findings, arthralgia and tonsil hypertrophy were not observed. She is not a smoker, but stands for long periods of time working as a hair stylist. She has no history of Raynaud's phenomenon, photosensitivity, or preceding upper respiratory infection.

No abnormality was identified by radiological examination, electrocardiograph or echocardiogram. Laboratory tests revealed mild abnormalities: slightly elevated C-reactive protein (CPR) $0.46 \mathrm{mg} / \mathrm{dl}$ (<0.3mg/dl), WBC 5620/ $\mathrm{LL}$, Neutrophils $72.5 \%$ $(37.0-72.0 \%)$ and elevated anti-streptolysin O (ASLO) levels 371.0 $\mathrm{IU} / \mathrm{ml}(<240 \mathrm{IU} / \mathrm{ml})$. Erythrocyte sedimentation rate was elevated to $28 \mathrm{~mm} / \mathrm{h}(3-15 \mathrm{~mm} / \mathrm{h})$. The complement activity $(\mathrm{CH} 50)>60.0 \mathrm{U} /$

Work submitted on 15.02.2016

Approved by the Advisory Board and accepted for publication on 19.06.2016

* Work performed at the Department of Dermatology, Mie University, Graduate School of Medicine - Mie, Japan.

Financial Support: None.

Conflict of Interests: None.

1 Department of Dermatology, Mie University Graduate School of Medicine - Mie, Japan. 
$\mathrm{ml}(23-46 \mathrm{U} / \mathrm{ml})$, serum IgG 1807.0mg/dl (895-1779mg/dl) and serum gamma globulin 21.6\% (10.5-20.3\%) were elevated. Antineutrophil cytoplasmic autoantibodies (P-ANCA, C-ANCA) were negative. Antinuclear antibody, anticardiolipin antibody, lupus anticoagulant, cryoglobulin and immune complex were undetectable. Hepatitis B surface antigen (HBsAg) and hepatitis C antibody were negative. Urinalysis was normal.

A skin biopsy specimen from a subcutaneous erythematous nodule of the thigh revealed necrotizing vasculitis (Figure 2). A medium sized muscular artery was surrounded by dense neutrophilic and mononuclear cell infiltration with eosinophil changes. The vascular

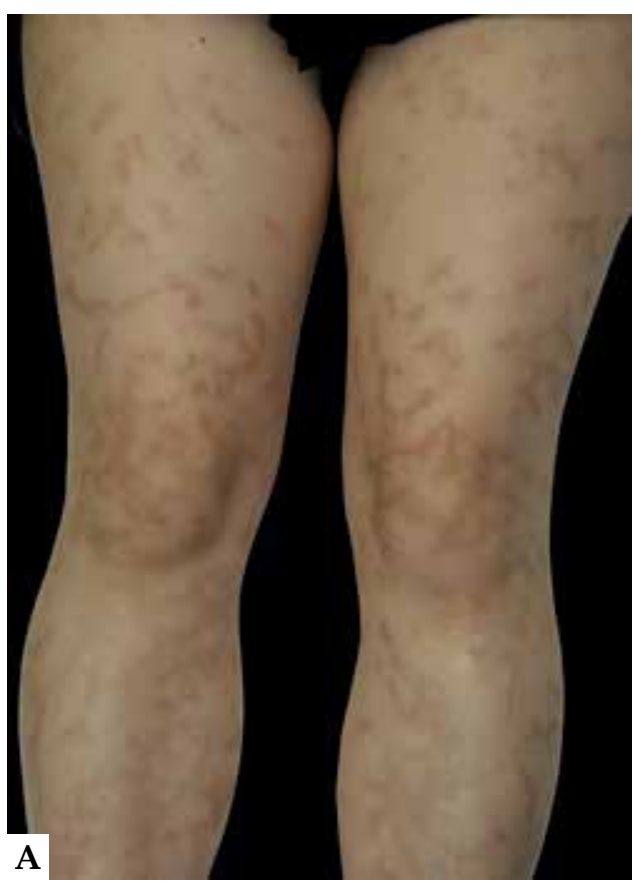

Figure 1:

Painful

net-like skin lesions were observed on all extremities on the first visit



Figure 3: Refractory skin depressions and pigmentation along the involved arteries remained as sequel wall was infiltrated by leukocytes and mononuclear cells with nuclear dust, fibrinoid degeneration, and the lumen was partially obliterated.

She was treated with aspirin $100 \mathrm{mg} /$ day and tocopherol nicotinate $600 \mathrm{mg} /$ day for a month without improvement. Livedo lesions progressed. Additional systemic prednisolone $10 \mathrm{mg} /$ day suppressed development of the new lesions and normalized CRP and serum gamma globulin levels. She has been well controlled for 18 months with prednisolone $5 \mathrm{mg}$ /day and aspirin $100 \mathrm{mg}$ /day, without relapse. However, many refractory skin depressions and pigmentation along the involved arteries remained as sequel (Figure 3).

\section{DISCUSSION}

CPAN is a benign form of polyarteritis nodosa restricted to the skin. ${ }^{1}$ There is a gender predominance of female to male of 1.7:1. ${ }^{2}$ The clinical manifestations of CPAN include tender multiple subcutaneous nodules, livedo reticularis, ulcers and inflammatory plaques on the extremities. Subcutaneous nodules are the most common findings (80-88\%), and livedo reticularis is also found in half of the patients. ${ }^{2-4}$ Palpable purpura is not a major skin manifestation of CPAN. The legs are affected in $97 \%$ of the patients, followed by the arms in $33 \%$, and the trunk in $8 \%{ }^{2}$

Presence of MPO-ANCA or PR3-ANCA is characteristic in ANCA related vasculitis (microscopic polyangiitis) and PAN. However, CPAN lacks specific serum markers. Therefore, histopathological diagnosis is indispensable for CPAN, and detection of necrotizing vasculitis in the small- to middle-sized arteries in the subcutis strongly suggests the diagnosis of CPAN. ${ }^{5}$ The early stage vascular lesions have more neutrophilic infiltration with nuclear dust. However, samples from a chronic lesion have more lymphocytic infiltrates in the vessels. Luminal occlusion with thrombi is also common in chronic lesions. ${ }^{6}$ Thus, these vascular changes induce ischemia of the surrounding dermal and subcutaneous tissue and cause skin depressions or ulcers. This case showed the characteristic small-artery vasculitis without systemic manifestations, strongly suggestive for CPAN.

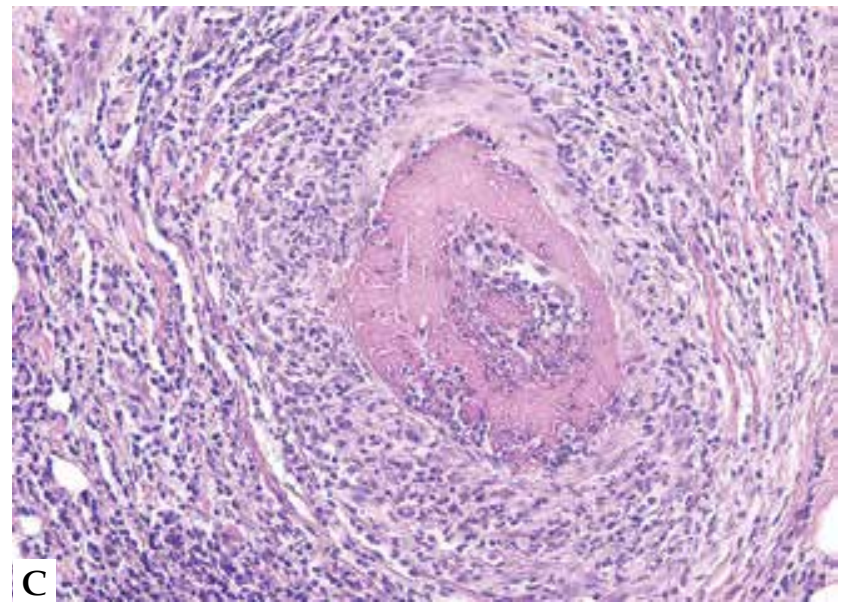

FigURE 2: Leukocytoclastic vasculitis in subcutis with infiltration of neutrophils and lymphocytes with nuclear dust, fibrin deposition and partial thrombotic occlusion was detected (Hematoxylin \& eosin stain X100) 
There is no consensus on the initial treatment of CPAN. In contrast to PAN and ANCA-related vasculitis, CPAN is a disease with favorable prognosis and less aggressive therapies are preferred. For a mild case of CPAN presenting with subcutaneous nodules without skin ulcers, nonsteroidal anti-inflammatory drugs, antiplatelet and anticoagulation therapies are indicated. For the treatment of acute severe cases with tender, necrotic, ulcerative skin lesions or extracutaneous organ involvement, systemic corticosteroid therapy is indicated. For resistant cases, additional azathioprine, dapsone, sulphapyridine, colchicine, and hydroxychloroquine are helpful. ${ }^{2}$ Because of no systemic organ involvement or acute aggressive skin lesions, the present case was treated with a milder antiplatelet therapy with- out effects. Immediately, we added systemic corticosteroid therapy that successfully controlled the vasculitis. However, many unsightly skin depressions and pigmented lesions remained on her arms and legs resulting in psychological distress. There was no report focusing on the remaining skin lesions after remission in patients with CPAN. The skin depressions are resistant to therapy and the pigmentation after inflammation is obvious in the exposed areas, especially in oriental skin. Unfortunately, these sequels can spread to the exposed areas, and impair one's quality of life. Herein we highlight the importance of early and aggressive treatment for CPAN to minimize the sequels, as well control of the disease activity.]

\section{REFERENCES}

1. Lindberg K. Ein Beitrag zur Kenntnis der Periarteritis nodosa. Acta Med Scand. 1931;76:183-225

2. Daoud MS, Hutton KP, Gibson LE. Cutaneous polyarteritis nodosa: a clinicopathological study of 79 cases. Br J Dermatol. 1997;136:706-13.

3. Kawakami T, Soma Y. Correlation of livedo racemosa, Cutaneous inflammatory plaques and antiphospholipid antibodies in patients with cutaneous polyarteritis nodosa. Medicine (Baltimore). 2011;90:119-24.

4. Morgan AJ, Schwartz RA. Cutaneous polyarteritis nodosa: a comprehensive review. Int J Dermatol. 2010;49:750-6.

5. Kawakami T. New algorithm (KAWAKAMI algorithm) to diagnose primary cutaneous vasculitis. J Dermatol. 2010;37:113-24

6. Elder DE, editor. Lever's histopathology of the skin: 10th ed. Philadephia: Lippincott Williams \& Williams; 2008. p. 517-9
MAILING ADDRESS:

Keiichi Yamanaka

2-174 Edobashi, Tsu, Mie 514-8507, Japan.

E-mail:yamake@clin.medic.mie-u.ac.jp

How to cite this article: Okada K, Nakamori R, Mizutani H, Yamanaka K. Cutaneous polyarteritis nodosa causing refractory skin deformation and pigmentation as sequel. An Bras Dermatol. 2017;92(5 Suppl 1): 53-5. 